\title{
Kinetics of inhibition in the biodegradation of monoaromatic hydrocarbons in presence of heavy metals
}

\author{
L Amor, C Kennes, M.C Veiga
}

Bioresource Technologye, Volume 78, Issue 2; 2001, Pages 181-5

DOI: 10.1016/S0960-8524(00)00182-6

\begin{abstract}
The toxicity and inhibitory effects of heavy metals such as cadmium, nickel and zinc on alkylbenzene removal were evaluated with a Bacillus strain. The kinetics of alkylbenzene biodegradation with the different heavy metals at various concentrations were modeled using the Andrews equation which yielded a good fit between model and experimental data. Additional experiments undertaken with a Pseudomonas sp. in presence of nickel confirmed a good fit between experimental data and the Andrews model for this strain as well. The heavy metals inhibition constants $\left(K_{\mathrm{i}}\right)$ were calculated for different combinations of volatile organic compounds (VOC) and heavy metals. The present approach provides a method for evaluating and quantifying the inhibition effect of heavy metals on the biodegradtion of pollutants by specific microbial strains.
\end{abstract}

\section{Keywords}

Biodegradation; Bioremediation; Toluene; Ethylbenzene; Xylene; Modeling; Toxicity; Heavy metals.

\section{Introduction}

Volatile organic compounds (VOC) which include the alkylated derivatives of benzene, such as toluene, ethylbenzene and xylenes are common environmental contaminants. They appear in gaseous and aqueous effluents of many industries as well as in contaminated soils and groundwaters. As a result of their presence in such ecosystems, they represent a serious threat to human health. Different alternatives are suitable for the removal of VOC. These alternatives include physical, chemical, as well as biological treatment technologies. The latter is characterized by its low treatment cost. At the same time biological processes minimize the environmental impact and residual contamination (Kovalick, 1991, Kovalick, 1992 and Skinner, 1991).

Since some contaminated systems contain both organic compounds and heavy metals, such as cadmium, zinc and nickel, the toxicity and inhibitory effects of heavy metals on microbial growth have received special attention. Metal ions contamination may inhibit microorganisms involved in the degradation of organic compounds and affect biodegradation rates. The level of inhibition will depend on the concentration and availability of the heavy metals and is dependent on the action of complex processes controlled by multiple factors including the nature of the metals, media and microbial 
species (De Rore et al., 1994, Goblenz et al., 1994, Hashemi et al., 1994, Olasupo et al., 1993 and Tomioka et al., 1994). Heavy metals inhibit microorganisms by blocking essential functional groups or interfering with essential metal ions incorporation of biological molecules (Doelman et al., 1994, Gadd and Griffiths, 1978, Li and Tan, 1994a, Li and Tan, 1994b and Wood and Wang, 1983). In some cases microorganisms are resistant to some heavy metals through different possible mechanisms (Rohit and Sheela, 1994 and Tomioka et al., 1994).

Studies have been published on the effect of heavy metals on bacterial growth (BenkaCoker and Ekundayo, 1998, Cabrero et al., 1998, Hassen et al., 1998a and Hassen et al., 1998b) but little has been reported on their effect on VOC biodegradation or on modeling such effects through simple classical kinetic equations. The goal of this study was to evaluate and model the effect of different concentrations of heavy metals, cadmium, nickel and zinc on the biodegradation of toluene, ethylbenzene and $o$-xylene by a Bacillus strain. Experiments were also undertaken with a Pseudomonas sp. in order to confirm, with a bacterial strain distinctly different from Bacillus sp., the good fit between the selected kinetic equation and the experimental data.

\section{Methods}

\subsection{Bacterial growth and medium}

The bacterial species used in this study were obtained from a biofilter treating air contaminated with volatile aromatic compounds (Kennes et al., 1996). They were grown in a liquid medium and were adapted to the toxic organic compounds and working conditions before the biodegradation assays. Isolation and taxonomic identification of the strains are described elsewhere (Veiga et al., 1999). Alkylbenzenes (toluene, ethylbenzene, o-xylene) mixture with over 99\% purity were used as a sole carbon source (Sigma, St. Louis, MO, USA). The biodegradation assays were performed with $5 \mathrm{ml}$ aqueous medium introduced in $50 \mathrm{ml}$ flasks closed with screw stoppers and viton septa.

The microorganisms were grown at $\mathrm{pH} 5.9 \pm 0.1$ in a medium containing: $\mathrm{KH}_{2} \mathrm{PO}_{4} 4.5$ $\mathrm{g} / \mathrm{l}, \mathrm{K}_{2} \mathrm{HPO}_{4} 0.50 \mathrm{~g} / \mathrm{l} ; \mathrm{NH}_{4} \mathrm{Cl} 2 \mathrm{~g} / \mathrm{l}$ and $\mathrm{MgSO}_{4} \cdot 7 \mathrm{H}_{2} \mathrm{O}_{0.10} \mathrm{~g} / \mathrm{l}$. Vitamins and trace minerals (Kennes et al., 1996) were also included. Media and materials were sterilized by autoclaving at $120^{\circ} \mathrm{C}$ for 20 min except for the vitamins and trace mineral solutions which were filter-sterilized and added to the medium after cooling down. The strains were then inoculated under sterile conditions. The inoculated medium was incubated at $30 \pm 1^{\circ} \mathrm{C}$ in a thermostated rotary-shaker. During the experiments the flasks were kept in the dark with constant shaking at 200 revolutions per minute. The inoculum was introduced using the same final biomass concentration in each flask. Finally the heavy metals and the alkylbenzenes were added. Concentrated stock solutions of the heavy metals (Sigma) were prepared in distilled water.

During the assays, samples were analyzed periodically. Duplicate or triplicate assays were performed in order to check the overall variability of the results. Control vials were prepared at the same time and consisted of flasks containing the same medium as for the assays with the compounds to be studied. Controls were inoculated and then autoclaved. Some assays without biomass (blanks) were incubated as well. Controls and 
blanks were used to check if any abiotic removal of the pollutants took place and to check for heavy metal precipitation in the absence of biomass.

\subsection{Analytical methods}

Alkylbenzenes were analyzed using a Hewlett Packard 5890 Series II gas chromatograph (Madrid, Spain) and a flame ionization detector (FID). Separation was achieved using a high resolution capillary TR-Wax column under isothermal conditions (Veiga et al., 1999). Concentrations of toluene, ethylbenzene and $o$-xylene were calculated by comparing the areas of standards to the areas and retention times of samples taken during the experiments. The alkylbenzene concentrations in the liquid phase were calculated according to Henry's law correlating equilibrium concentrations between the gas and the liquid phase. Since heavy metals could partly precipitate in the aqueous medium used in the assays, their initial concentration was checked by atomic absorption spectrophotometry on a Varian SpectrAA 300/400 (Madrid, Spain).

\section{Results and discussion}

\subsection{Effect of heavy metals on alkylbenzene biodegradation}

Preliminary assays were performed to evaluate the most suitable heavy metal concentration range to be used for each metal in this work (data not shown). The heavy metal concentrations added in the assays described below corresponded to the following range: $\mathrm{Zn}$ 0.04-0.47 $\mathrm{mM}$, Ni $0.4-1.0 \mathrm{mM}$; Cd $0.33-1.13 \mathrm{mM}$, although the concentrations tested depended also on the type of alkylbenzene and microbial strain.

In Fig. 1 typical alkylbenzene biodegradation data are shown for Bacillus sp. and for Pseudomonas sp. in the absence of heavy metals. Based on these results it is apparent that both cultures were able to degrade the compounds although $o$-xylene was minimally degraded by Pseudomonas sp. When experiments were initiated with a mixture of all three contaminants at similar concentrations, Bacillus initially degraded toluene followed by ethylbenzene and finally o-xylene. Conversely, Pseudomonas initially degraded ethylbenzene then toluene and finally the xylene isomer. Alkylbenzene concentrations remained basically constant in autoclaved control bottles and in non-inoculated vials. Although the biodegradation of alkylbenzenes by Pseudomonas strains has been reported in several studies (Chang et al., 1993, Choi et al., 1992 and Pedersen et al., 1997) available data regarding Bacillus strains are quite minimal ( Natarajan et al., 1994 and Uma and Sandhya, 1998). The higher recalcitrance of xylene isomers in alkylbenzene mixtures has been observed by some groups (Alvarez and Vogel, 1991 and Anid et al., 1993). 

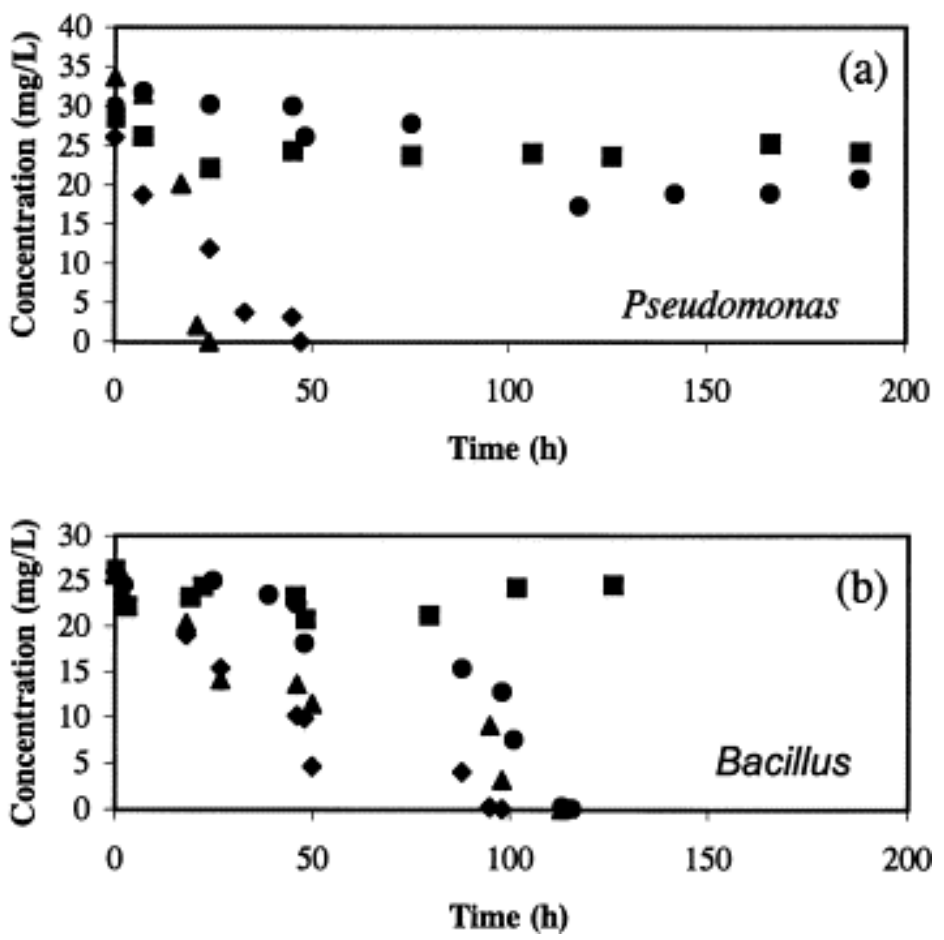

Fig. 1.

Toluene $(\bullet)$, ethylbenzene $(\boldsymbol{\Delta})$ and $\boldsymbol{o}$-xylene $(\bullet$ ) biodegradation profiles for: (a) Pseudomonas sp. and (b) Bacillus sp. in the absence of heavy metals. Control ( $\square$ ) is only represented for one compound (toluene) in order not to overload the figure.

It has been reported that a given aromatic compound may sometimes inhibit the biodegradation of another one available in the medium, when more than one VOC is present. Evans et al. (1991) observed that the depletion of $o$-xylene was correlated with and dependent on the metabolism of toluene. Chang et al. (1993) showed that toluene had a greater effect on the degradation of $p$-xylene than $p$-xylene had on toluene degradation working with a Pseudomonas sp. Such inhibition phenomenon may also be expected with heavy metals such as cadmium, nickel or zinc. An example of inhibition levels in toluene biodegradation obtained with Bacillus sp. in presence of the different heavy metals is shown in Fig. 2. Similar data (not shown) were observed with the other alkylbenzenes. In all cases the removal rates were higher without metals in the medium showing the inhibitory effect of the heavy metals on biodegradation of VOC even at low concentrations. These results indicated that alkylbenzene biodegradation was already partly inhibited at relatively low heavy metal concentrations. However, other researchers ( Hassen et al., 1998b) studying the influence of heavy metals on microbial growth rate observed that zinc at concentrations between 0.05 and $0.2 \mathrm{mM}$ did not have substantial effects on the growth of Bacillus thuringensis. In another study ( Hassen et al., 1998a) it was found that the growth rate of most Cd-tolerant strains was essentially not affected by Cd concentrations below $1.5 \mathrm{mM}$. These authors examined several pure cultures including Bacillus thurigensis, Pseudomonas paucimobilis, Providencia rettgeri, Proteus mirabilis, Klebsiella rhinoscleromatis, Citrobacter freundii and Escherichia coli K12 and observed that the most tolerant strain was a strain of Pseudomonas aeruginosa. In the present study inhibition of alkylbenzene removal appeared at lower concentrations irrespective of the alkylbenzene used. Inhibition at such concentrations or even at lower ones was also observed by Benka-Coker and 
Ekundayo (1998). Nickel appeared to be the least toxic of all three heavy metals. This was in agreement with other recent studies showing that at low concentrations nickel was nontoxic and actually enhanced growth rate and metabolic activity of aerobic or anaerobic bacteria ( Dilek et al., 1998 and Gonzalez-Gil et al., 1999). Zinc exhibited the highest toxicity and was the only heavy metal showing already partial inhibition at concentrations near $0.05 \mathrm{mM}$. It has also been observed that zinc was inhibitory to a Pseudomonas sp. at concentrations below $0.01 \mathrm{mM}$ ( Benka-Coker and Ekundayo, 1998).

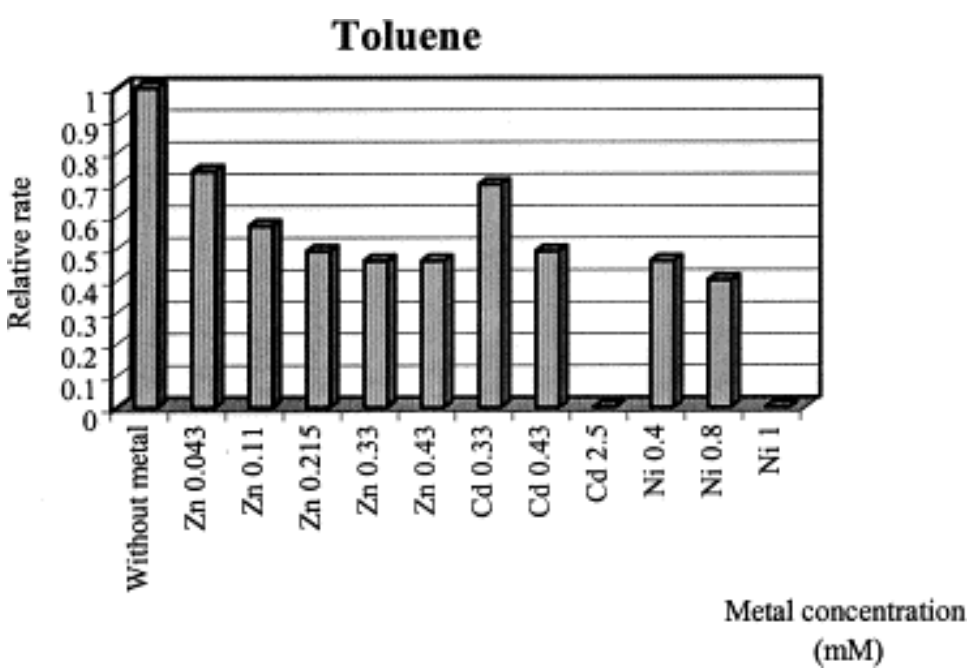

Fig. 2.

$(\mathrm{mM})$

Levels of inhibition of toluene biodegradation for Bacillus sp. with and without heavy metals, taking the data obtained without heavy metal as a reference (no inhibition).

\subsection{Modeling kinetics of toluene, ethylbenzene and o-xylene removal in presence of heavy metals}

Strains able to grow on aromatic VOC usually show either zero or first-order growth kinetics depending on the substrate concentration, which can be modeled by using the Monod equation. If inhibition is observed at higher VOC concentrations an additional inhibition term has to be added leading to a new equation (Andrews, 1968).

Compounds other than the primary carbon source may also affect growth or biodegradation rates. In that sense heavy metals could play such a role. Heavy metals are often added at low concentrations in the form of trace mineral solutions to microbial culture media to maintain or enhance microbial growth. Zinc and nickel are also components of several bacterial enzymes. It would therefore not be surprising to observe variable growth rates and substrate removal rates with varying heavy metal concentrations, which could either present a favorable effect or an inhibitory effect depending on their concentration. Nickel, for example, is known to enhance the activity of some anaerobic bacteria under given culture conditions (Gonzalez-Gil et al., 1999) while inhibition is observed at higher concentrations (Lin and Chen, 1997). A similar behavior is expected with other bacterial groups. Indeed, other experiments undertaken with activated sludge (Dilek et al., 1998) demonstrated that microbial growth rates may significantly be enhanced at nickel and chromium concentrations around $0.01 \mathrm{mM}$ while higher concentrations can be inhibitory. In the present work, the heavy metals 
added to the medium were only added at inhibitory concentrations. No enhancements of growth rates or biodegradation rates were observed at the lowest concentrations tested.

Different kinetic equations were tested with data obtained during the studies with Bacillus sp. and with Pseudomonas sp. in order to quantify the effect of each heavy metal on biodegradation of alkylbenzenes. Although the heavy metals are not the growth substrates, the effect on substrate (toluene, ethylbenzene and $o$-xylene) removal kinetics with increasing heavy metals concentrations could be modeled mathematically using the Andrews model ( Andrews, 1968). This model is usually used for quantifying microbial growth rates with inhibition but was considered to be an appropriate model since alkylbenzene removal rates appeared to be directly linked to microbial growth response for both strains used in this study ( Fig. 3), although reports describing this application of the kinetic equation on media containing heavy metals are minimal.
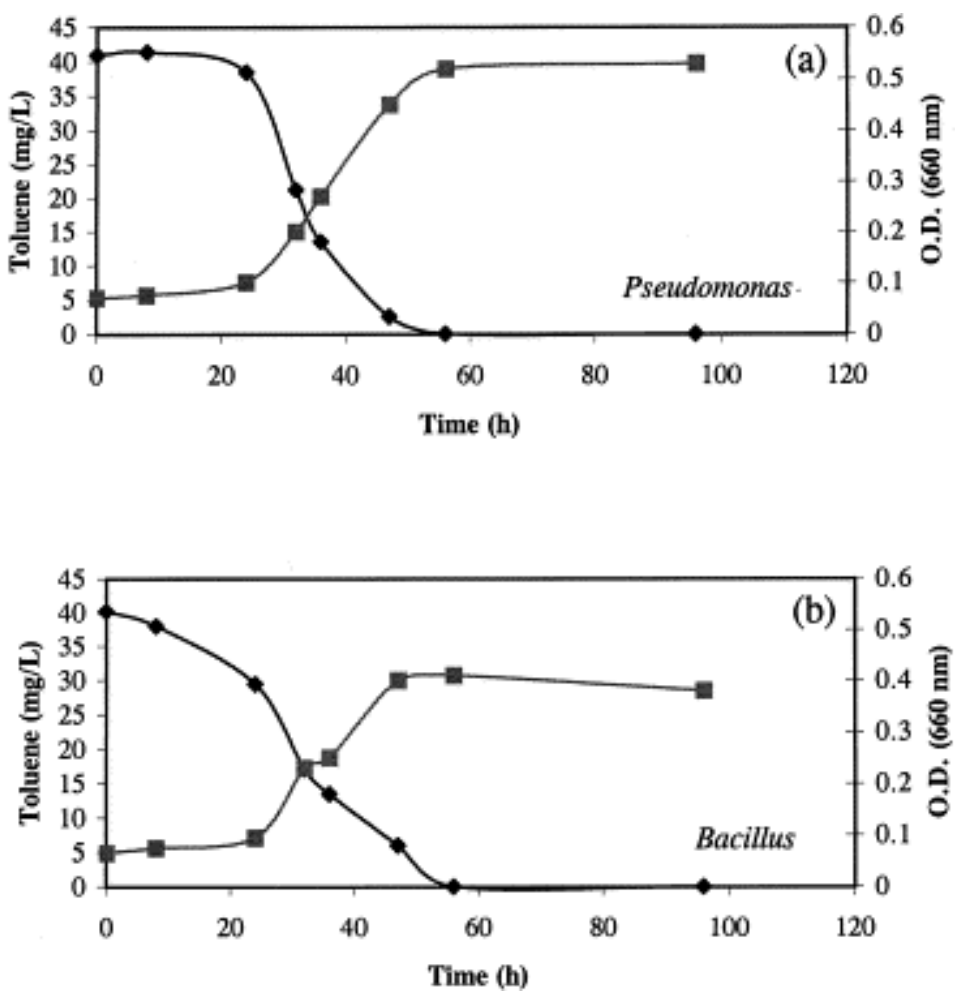

Fig. 3.

Biodegradation pattern ( $\downarrow$ ) versus growth pattern (๘) for: (a) Pseudomonas sp. and (b) Bacillus sp.

The heavy metals inhibition constants $\left(K_{\mathrm{i}}\right)$ for VOC removal were calculated according to the equation:

$r=r_{\max } \frac{S}{K_{\mathrm{s}}+S+\left(S^{2} / K_{\mathrm{i}}\right)}$,

where $r_{\max }$ is the maximum toluene, ethylbenzene or $o$-xylene degradation rate, $S$ is the heavy metal concentration and $K_{\mathrm{s}}$ represents a substrate concentration called the Monod constant. However, since in the heavy metal concentration range tested only inhibitory effects were observed, the equation was simplified by considering that $K_{\mathrm{s}}$ was negligible $(S \gg K)$ : 
$\frac{1}{r}=\frac{1}{r_{\max }}+\frac{S}{K_{\mathrm{i}} r_{\max }}$

In such cases, a plot of (degradation rate) ${ }^{-1}$ versus heavy metal concentrations should yield a straight line. The experimental data obtained with Bacillus conformed with Andrews kinetics when plotting the reciprocal of degradation rate versus heavy metal concentrations. An example is presented for Bacillus in Fig. 4. Additional experiments were also undertaken with the Pseudomonas sp. in presence of nickel in order to confirm the good fit between model and experimental data with a distinctly different bacterial species. In all cases, $R^{2}$ values ranged between 0.90 and 1.0. In Table 1 the $K_{\mathrm{i}}$ values are shown for the different alkylbenzenes for both strains. The results presented in Table 1 confirmed the data and conclusions obtained from biodegradation curves and removal rates.

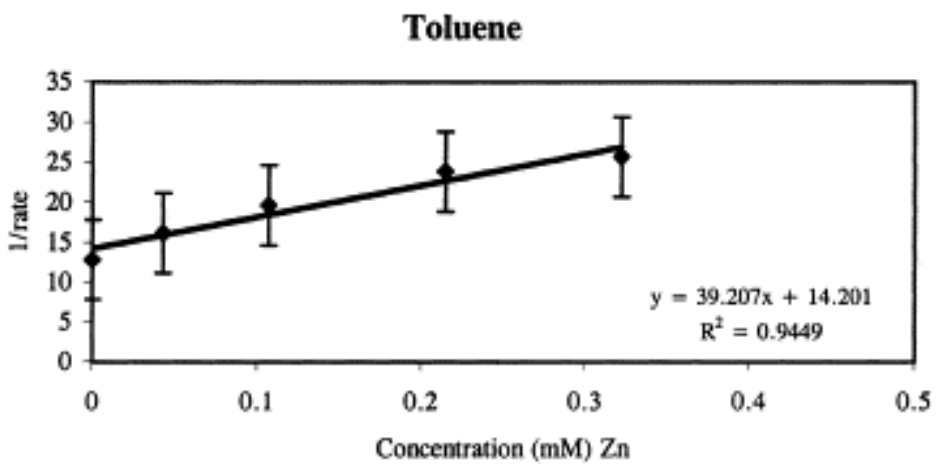

Fig. 4.

Typical reciprocal plot of rate versus heavy metal concentrations obtained with toluene and Bacillus sp.

Table 1.

Inhibition constants $\left(k_{\mathrm{i}}, \mathrm{mM}\right)$ for both strains with the different VOC and heavy metals tested

\begin{tabular}{|c|c|c|c|c|c|}
\hline \multirow[b]{2}{*}{ Toluene } & \multicolumn{2}{|c|}{ Bacillus sp. } & \multicolumn{2}{|c|}{ Pseudomonas sp. } & $\begin{array}{l}\text { p. } \\
\text { Nicke }\end{array}$ \\
\hline & 0.448 & 0.4030 .606 & 0.339 & 0.041 & 0.662 \\
\hline Ethylbenzene & 0.757 & 0.4200 .690 & $\mathrm{ND}^{\mathrm{a}}$ & ND & ND \\
\hline$o$-xylene & 0.416 & 0.4310 .620 & ND & ND & ND \\
\hline
\end{tabular}

\section{Conclusions}

Biodegradation experiments showed that nickel was in most cases the least toxic heavy metal for alkylbenzene degradation. Conversely, zinc was usually the most toxic heavy metal for alkylbenzene removal. The experimental results could be modeled with the Andrews equation providing a quantitative estimation of the inhibition effect.

\section{Acknowledgements}


This research was supported by project AMB 98-0288. We thank Endesa and Repsol Petróleo (La Coruña, Spain) for their collaboration in this study, mainly with heavy metal analysis.

\section{References}

\section{P.J.J Alvarez, T.M Vogel}

Substrate interactions of benzene, toluene and para-xylene during microbial degradation by pure cultures and mixed culture aquifier slurries

Appl. Environ. Microbiol., 57 (1991), pp. 2981-2985

\section{J.F Andrews}

A mathematical model for the continuous culture of microorganisms utilizing inhibiting substrates

Biotechnol. Bioeng., 10 (1968), pp. 707-723

P.J Anid, P.J.J Alvarez, T.M Vogel

Biodegradation of monoaromatic hydrocarbons in aquifer columns amended with hydrogen peroxide and nitrate

Water Res., 27 (1993), pp. 685-691

M.O Benka-Coker, J.A Ekundayo

Effects of heavy metals on growth of species of Micrococcus and Pseudomonas in a crude oil/mineral salts medium

Bioresource Technol., 66 (1998), pp. 241-245

A Cabrero, S Fernández, F Mirada, J García

Effects of copper and zinc on the activated sludge bacteria growth kinetics

Water Res., 32 (1998), pp. 1355-1362

M Chang, T.C Voice, C.S Criddle

Kinetics of competitive inhibition and cometabolism in the biodegradation of benzene, toluene and $p$-xylene by two Pseudomonas isolates

Biotechnol. Bioeng., 41 (1993), pp. 1057-1065

Y.-B Choi, J.-Y Lee, H.-S Kim

A novel bioreactor for the biodegradation of inhibitory aromatic solvents: experimental results and mathematical analysis

Biotechnol. Bioeng., 40 (1992), pp. 1403-1411

H De Rore, E Tup, F Houwen, M Mergeay, W Verstraete

Evolution of heavy metal resistant transconjugants in a soil environment with a concomitant selective press

FEMS Microbiol. Ecol., 14 (1994), pp. 263-273

F.B Dilek, C.F Gokcay, U Yetis

Combined effects of $\mathrm{Ni}(\mathrm{II})$ and $\mathrm{Cr}(\mathrm{VI})$ on activated sludge

Water Res., 2 (1998), pp. 303-312 
P Doelman, E Jansen, M Michels, M Van Til

Effects of heavy metals in soil on microbial diversity and activity as shown by the sensitivity-resistance index, an ecologically relevant parameter

Biol. Fert. Soils, 17 (1994), pp. 177-184

P.J Evans, D.T Mang, L.Y Young

Degradation of toluene and $m$-xylene and transformation of $o$-xylene by denitrifying enrichment cultures

Appl. Environ. Microbiol., 57 (1991), pp. 450-454

G.M Gadd, A.J Griffiths

Microorganism and heavy metals

Microbiol. Ecol., 4 (1978), pp. 303-317

A Goblenz, K Wolf, P Banda

The role of gluthathione biosynthesis in heavy metal resistance in the fission yeast Schizosaccharomyces pombe. Metals and microorganisms: relationships and applications

FEMS Microbiol. Rev., 14 (1994), pp. 303-308

\section{Gonzalez-Gil et al., 1999}

G Gonzalez-Gil, R Kleerebezem, G Lettinga

Effects of nickel and cobalt on kinetics of methanol conversion by methanogenic sludge as assessed by on-line $\mathrm{CH}_{4}$ monitoring

Appl. Environ. Microbiol., 65 (1999), pp. 1789-1793

F Hashemi, G.G Leppard, D.J Kushner

Copper resistance in Anabaena variabilis: effects of phosphate nutrition and polyphosphate bodies

Microbiol. Ecol., 27 (1994), pp. 159-176

Hassen et al., 1998a

A Hassen, N Saidi, M Cherif, A Boudabous

Resistance of environmental bacteria to heavy metals

Bioresource Technol., 64 (1998), pp. 7-15

A Hassen, N Saidi, M Cherif, A Boudabous

Effects of heavy metals on Pseudomonas aeruginosa and Bacillus thuringiensis

Bioresource Technol., 65 (1998), pp. 73-82

C Kennes, H Cox, M Doddema, W Harder

Design and performance of biofilters for the removal of alkylbenzene vapors

J. Chem. Technol. Biotechnol., 66 (1996), pp. 300-304

W.W Kovalick Jr.

Trends in innovative treatment technologies at contaminated sites

Water Sci. Technol., 26 (1992), pp. 99-106 
Kovalick Jr., W.W., 1991. In: Sayler, G.S., Fox, R., Blackburn, J.W. (Eds.), Removing Impediments to the Use of Bioremediation and other Innovative Technologies in Environmental Biotechnology for Waste Treatment, vol. 41. Plenum Press, New York, pp. 53-58

F Li, T.C Tan

Effect of heavy metals ions on the efficacy of a mixed bacilli BOD sensor

Biosens. Bioelectron., 9 (1994), pp. 315-324

F Li, T.C Tan

Monitoring BOD in the presence of heavy metals ions using a poly (4-vinylpyridine) coated microbial sensor

Biosens. Bioelectron., 9 (1994), pp. 445-455

C.-Y Lin, C.-C Chen

Toxicity-resistance of sludge biogranules to heavy metals

Biotechnol. Lett., 19 (1997), pp. 557-560

M.R Natarajan, Z Lu, P Oriel

Cloning and expression of a pathway for benzene and toluene from Bacillus stearothermophilus

Biodegradation, 5 (1994), pp. 77-82

N.A Olasupo, M.B Scott-Emuakpor, R.A Ogunshola

Resistance to heavy metals by some Nigerian yeast strain

Folia Microbiologica, 38 (1993), pp. 285-287

A.R Pedersen, S Moller, S Molin, E Arvin

Activity of toluene-degrading Pseudomonas putida in the early growth phase of a biofilm for waste gas treatment

Biotechnol. Bioeng., 54 (1997), pp. 131-141

M Rohit, S Sheela

Uptake of zinc in Pseudomonas sp. strain UDG26

Appl. Environ. Microbiol., 60 (1994), pp. 2367-2370

Skinner, 1991

Skinner, J.H., 1991. Bioremediation research issues. In: Sayler, G.S., Fox, R., Blackburn, J.W. (Eds.), Environmental Biotechnology for Waste Treatment, vol. 41. Plenum Press, New York, pp. 61-70

Tomioka et al., 1994

N.A Tomioka, H Uchiyama, O Yagi

Cesium accumulation and growth characteristics of Rhodococcus erythropolis CS98 and Rhodococcus sp. strain CS402

Appl. Environ. Microbiol., 60 (1994), pp. 2227-2231 
B Uma, S Sandhya

Kinetics of pyridine degradation along with toluene and methylene chloride with Bacillus sp. in packed bed reactor

Bioprocess Eng., 18 (1998), pp. 303-305

M.C Veiga, M Fraga, L Amor, C Kennes

Biofilter performance and characterization of a biocatalyst degrading alkylbenzene gases

Biodegradation, 10 (1999), pp. 169-176

J.M Wood, H.K Wang

Microbial resistance to heavy metals

Environ. Sci. Technol., 17 (1983), pp. 582-590 